\title{
Quantum nondemolition and higher order effects for a nonlinear meter in an interferometric gravitational wave antenna
}

\author{
Yu. Levin \\ Theoretical Astrophysics, California Institute of Technology, Pasadena, California 91125
}

(Received 11 March 1997; published 12 January 1998)

\begin{abstract}
A new optical topology and signal readout strategy for a laser interferometer gravitational wave detector were proposed recently by Braginsky and Khalili. Their method is based on using a nonlinear medium inside a microwave oscillator to detect the gravitational-wave-induced spatial shift of the interferometer's standing optical wave. This paper proposes a quantum nondemolition scheme that could be realistically used for such a readout device and discusses a "fundamental" sensitivity limit imposed by a higher order optical effect. [S0556-2821(97)06222-X]
\end{abstract}

PACS number(s): 04.80.Nn, 05.40.+j, 06.20.Dk

\section{INTRODUCTION AND SUMMARY}

Laser interferometer gravitational wave detectors (LIGO, VIRGO, GEO 600, TAMA) are designed to detect small perturbations $h$ in the spatial metric due to gravitational waves (GW's) passing through the Earth [1]. Being very far from major astrophysical sources [2], these detectors are likely to encounter GW's that are very weak, so the detectors must be correspondingly sensitive-e.g., the first LIGO interferometer will be able to detect GW's with $h \sim 3 \times 10^{-21}$ in the frequency band of $30-300 \mathrm{~Hz}$. Improving the sensitivity of measurement may be necessary to achieve the first GW detection and will surely be necessary to improve the event rate.

One of the major noise sources in traditional interferometers is the so-called shot noise. What is being detected is the phase shift of the output optical wave [1]:

$$
\delta \phi \sim \omega_{\mathrm{opt}} \tau_{\mathrm{GW}} h
$$

where $\omega_{\text {opt }}$ is the angular frequency of the optical wave and $\tau_{\mathrm{GW}}$ is the half-period of a gravitational wave. For coherent optical pumping the uncertainty in the phase due to shot noise is given by $\Delta \phi=1 / \sqrt{N_{\mathrm{GW}}}$, where $N_{\mathrm{GW}}$ is the number of photons introduced into the interferometer during $\tau_{\mathrm{GW}}$. Thus a gravitational wave can be detected if

$$
N_{\mathrm{GW}}>N_{\mathrm{min}} \simeq \frac{1}{\left(h \omega_{\mathrm{opt}} \tau_{\mathrm{GW}}^{*}\right)^{2}}
$$

Therefore, in order to increase the gravity wave sensitivity of the interferometer, we have to increase the number of photons in the resonator (and hence the consumed laser power) as $N \propto 1 / h^{2}$. On the other hand, the presence of the large number of optical photons in the resonator poses severe technical and fundamental problems. Among the technical problems are distortion of mirrors due to overheating, and large laser power consumption [1]. The fundamental problem is that photons in the interferometer will randomly buffet the mirrors inducing random motion indistinguishable from the motion produced by a gravitational wave. Balancing this ra- diation pressure noise and the shot noise produces the standard quantum limit (SQL) for monitoring the displacements of the test masses [2].

Recently Braginsky and Khalili have proposed a new way to improve the sensitivity of an interferometric GW detector without increasing the interferometer's optical power [3]. Their method entails a new type of GW readout based on a microwave oscillator containing an optically nonlinear medium, which is placed inside the GW detector's high quality Fabry-Perot (FP) resonator. The advantage of this readout method is that, unlike conventional interferometers, it does not require large optical power circulating inside the FP resonator in order to achieve high sensitivity. In Sec. II the principles of this scheme are briefly outlined and some numerical estimates are quoted.

Section III describes a potentially practical quantum nondemolition (QND) strategy which can be used in the Braginsky-Khalili (BK) readout system. We show in Sec. III and Appendix A [cf. Eq. (21)] that a QND measurement can be performed within a narrow frequency band centered around

$$
\Omega_{0}=\sqrt{\frac{6 \hbar N}{m c L}} \omega_{\mathrm{opt}},
$$

where $\omega_{\text {opt }}$ and $N$ are the frequency of light and the number of photons stored in the FP resonator, respectively, $L$ is the distance between the end mirrors of the FP resonator and $m$ is the mass of each of the test masses to which the mirrors are attached. For $N=2.8 \times 10^{20}, L=4 \mathrm{~km}, m=10 \mathrm{~kg}$, $\omega_{\text {opt }}=3 \times 10^{15}$ one obtains $\Omega_{0} / 2 \pi=60 \mathrm{~Hz}$, which is within LIGO band. For the resonator's relaxation time of $10 \mathrm{~s}$ (as assumed in [3]) the necessary laser power to achieve this number of photons inside the resonator is $\sim 9 \mathrm{~W}$.

We demonstrate in Appendix B that the bandwidth $\Delta \Omega$ of this measurement determines the optimal power input to the microwave oscillator:

$$
W_{\text {optimal }} \sim W_{\mathrm{SQL}} \frac{\Omega_{0}}{\Delta \Omega},
$$

where $W_{\mathrm{SQL}}$ is the power input necessary to achieve the standard quantum limit sensitivity at frequency $\Omega_{0}$; cf. Eq. 


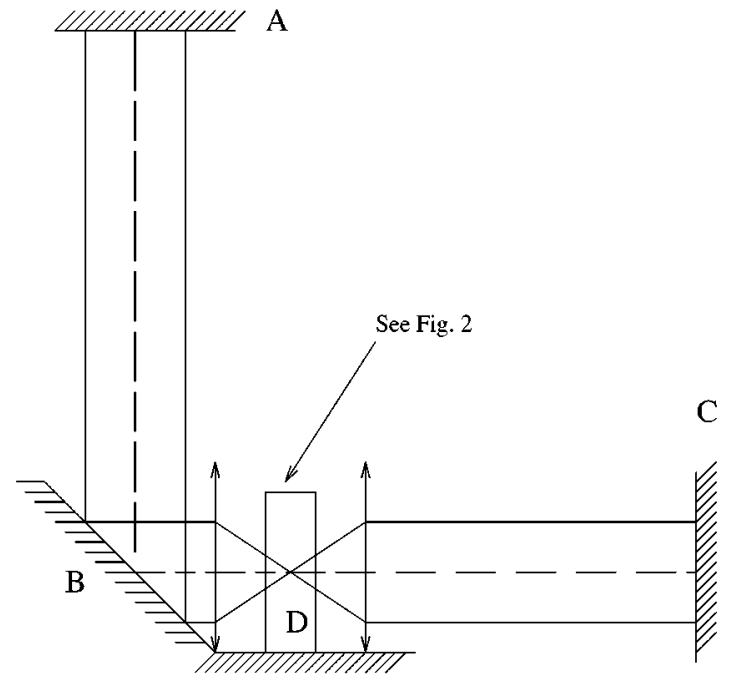

FIG. 1. The BK readout system. Two thin lenses focus light on a block $D$ containing a nonlinear medium. Block $D$ is shown in Fig. 2.

(24) where the expression and the numerical estimate for $W_{\mathrm{SQL}}$ are given. The signal-to-noise ratio achieved by this QND measurement is greater by a factor of $\sqrt{\Omega_{0} / \Delta \Omega}$ than the SQL:

$$
\left(\frac{S}{N}\right)_{\mathrm{QND}} \sim \sqrt{\frac{\Omega_{0}}{\Delta \Omega}}\left(\frac{S}{N}\right)_{\mathrm{SQL}} .
$$

Section IV and Appendix C discuss a higher order optical effect in the BK readout system and derive the sensitivity limit that it imposes. In particular, thermally excited mechanical modes in the test masses will, after interacting with light inside the FP resonator, produce a "double conversion" of photons, which will be registered as noise by the detector; cf. Eq. (25) and Eq. (26).

\section{PRINCIPLE OF OPERATION OF THE BK METER}

The layout of the BK meter is shown in Fig. 1 (for more details the reader is referred to [3]). Three freely suspended mirrors- $A, B$, and $C$-form walls of an $L$-shaped FabryPerot (FP) resonator which supports a standing optical wave, driven by a laser at end $A$ or $C$. Section $A-B$ of the resonator would be in one arm of the LIGO (or other) vacuum system, and $B-C$ in the other. The block $D$ containing two thin slabs of nonlinear medium (Fig. 2) is sandwiched between two thin focusing lenses two focal lengths apart. The lenses and the block are attached to mirror $B$.

When the polarization tensor of a gravitational wave is aligned with the arms of the FP resonator, the distances between $A$ and $B$ and between $B$ and $C$ will change in counterphase, i.e., when one is increasing, the other one will decrease. This will produce the a net spatial shift of the standing optical wave with respect to mirror $B$, thus changing the amplitude of the optical field within the two slabs of nonlinear medium. The slabs have cubic nonlinearities that are equal in magnitude but opposite in sign. They are positioned symmetrically with respect to the crest of the standing optical wave as shown in Fig. 2. Block $D$, which contains the

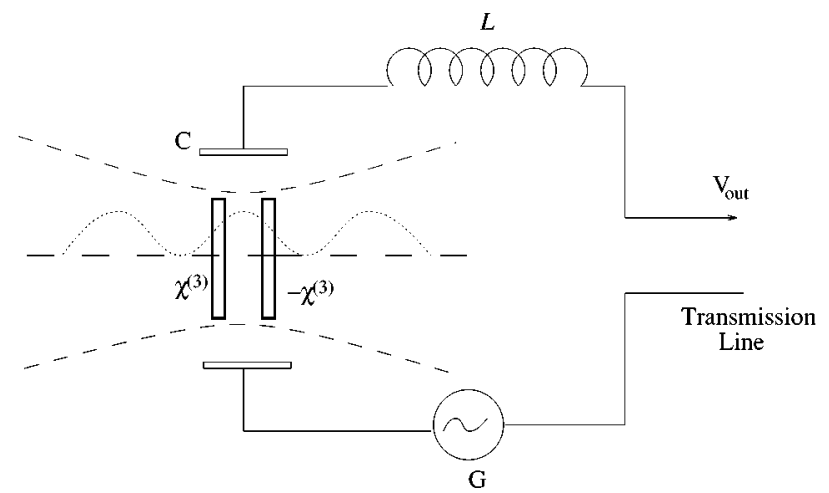

FIG. 2. This is an enlarged view of block $D$ from Fig. 1. Two slabs of nonlinear medium are positioned at the points of the maximal gradient of the intensity of the optical standing wave. The spatial shift of the optical standing wave changes the resonant frequency of the microwave $L C$ oscillator.

slabs, is placed in between the plates of a capacitor which in turn is part of a microwave oscillator.

The spatial shift in the optical standing wave produces changes of electric field in the first and second slabs that are equal in magnitude and opposite in sign:

$$
\delta E_{1}=-\delta E_{2} .
$$

Since the two slabs have the opposite nonlinearities, $\chi_{1}^{(3)}=-\chi_{2}^{(3)}$, the change in the index of refraction is the same for both of them:

$$
\delta n_{1}=\delta n_{2}=4 \pi \chi_{1}^{(3)} E_{1} \delta E_{1} .
$$

This change in dielectric constants of the plates in turn changes the value of the microwave oscillator's capacitance, thus producing a shift in its resonant frequency:

$$
\delta \omega_{e}=\frac{K \omega_{\mathrm{opt}}}{2} h,
$$

where $\omega_{e}$ is the frequency of the microwave oscillator, $\omega_{\mathrm{opt}}$ is the frequency of the optical wave, and $K=16 \pi^{2} \chi^{(3)} l N \hbar \omega_{\mathrm{opt}} \omega_{e} / V c$. Here $l$ is the width of each of the nonlinear slabs and $V$ is the volume of the capacitor. This shift is seen as a phase shift in the readout of the microwave oscillator:

$$
\delta \phi=\delta \omega_{e} \tau_{e}^{*}=\frac{1}{2} K \omega_{\mathrm{opt}} \tau_{e}^{*} h,
$$

where $\tau_{e}^{*}$ is the oscillator's ringdown time.

Braginsky and Khalili compare this with the traditional optical readout schemes in which the phase shift of the recombined optical wave is detected:

$$
\delta \phi_{\mathrm{opt}}=\omega_{\mathrm{opt}} \tau_{0}^{*} h,
$$

where $\tau_{0}^{*}$ is the ringdown time of the two traditional FP resonators, one in each arm of the interferometer. For $\chi^{(3)}=10^{-14} \mathrm{~cm}^{2} / \mathrm{V}^{2}$ (fused silica), $E_{\mathrm{opt}}^{2}=10^{7} \mathrm{~V}^{2} / \mathrm{cm}^{2}$ (optical breakdown of fused silica) they calculate $K$ in Eq. (8) to 
be of order 1 , so for $\tau_{\mathrm{opt}}^{*} \sim \tau_{e}^{*}$ the responses of both systems in terms of phase shift are of the same order:

$$
\phi_{\mathrm{opt}} \sim \phi_{e}
$$

For coherent pumping in both cases the uncertainty in the phase is $\Delta \phi \sim 1 / \sqrt{N_{\mathrm{GW}}}$, where $N_{\mathrm{GW}}$ is the number of photons (optical in conventional interferometers and microwave in the BK readout system) introduced into the interferometer during an averaging time (half the GW period). So to achieve the same level of sensitivity one needs to pump the same number of photons in both cases, but the power needed by the BK meter is smaller by a factor of $\omega_{\text {opt }} / \omega_{e} \sim 10^{4}$. The BK estimate for the microwave power is

$$
W_{e}=\frac{\hbar \omega_{e} N_{e}}{\tau_{e}^{*}} \sim 1 \mathrm{~W}
$$

for $N_{e} \sim 10^{20}$. For more detailed estimates the reader is referred to Ref. [3].

\section{QND FOR THE BK READOUT SYSTEM}

Any readout system that monitors the displacement of the mirrors must exert on them a fluctuating back action force, thus enforcing the Heisenberg uncertainty relation. As a consequence of this, all straightforward displacement measurements run into the standard quantum limit (SQL) $[4,5]$

$$
\Delta x_{\mathrm{SQL}}=\sqrt{\frac{\hbar \tau}{m}}
$$

where $\Delta x$ is the minimal uncertainty in displacement of a free mass $m$ monitored over a time interval $\tau$. This SQL for displacement can also be written in terms of the limiting spectral density of the mirrors' displacement fluctuations [4]:

$$
S_{x}^{\mathrm{SQL}}(\Omega)=\frac{\hbar}{m \Omega^{2}},
$$

where $\Omega$ is the frequency. Then $\Delta x=\sqrt{S_{x}^{S Q L} \Delta \Omega}$, and for $\Omega \sim \Delta \Omega \sim 1 / \tau$ one recovers Eq. (13).

The SQL for a free mass is by no means a fundamental limit; it can be overcome by a variety of techniques [6] which are known collectively as quantum nondemolition (QND) measurements. All previously proposed QND schemes that are applicable for conventional GW interferometers utilize highly nonclassical states of light, and none of them are practical because of technical difficulties (most especially because of the large required optical pumping power and because losses so easily destroy the nonclassical states of light). In this paper a different strategy is proposed, one which does not require the deliberate creation or detection of any nonclassical state of light and thus can be more practically implemented. This scheme, however, is confined to narrow-band measurements.

We begin by describing the back action mechanism by which the BK readout system enforces the Heisenberg uncertainty relation on the measurement of the test-mass position. The quantum state of the BK microwave oscillator satisfies the usual phase-number uncertainty relation

$$
\Delta \phi_{e} \Delta N_{e}>\frac{1}{2}
$$

The more accurately the BK meter reads out $\phi_{e}$, the larger will be the fluctuations $\Delta N_{e}$ in the oscillator's number of microwave photons. The $\chi^{(3)}$ nonlinearity will transform $\Delta N_{e}$ into an uncertainty of the optical index of refraction of the slabs:

$$
\delta n_{1}=-\delta n_{2}=\frac{16 \pi^{2} \chi^{(3)} \hbar}{\epsilon V} \delta N_{e},
$$

where $\delta N_{e}$ is the fluctuation in $N_{e}, \delta n_{1}$ and $\delta n_{2}$ are the resulting fluctuations in $n_{1}$ and $n_{2}, \epsilon$ is the coefficient of dielectric permittivity, and $V$ is the volume of the capacitor. Braginsky and Khalili have argued [3] that $\delta n_{1}$ and $\delta n_{2}$ cause a redistribution of the optical energy between the left and the right parts of the FP resonator, thereby giving rise to a net difference in the forces buffeting the mirrors:

$$
\delta F=K \frac{\omega_{\mathrm{opt}}}{\omega_{e}} \frac{\hbar \delta N_{e}}{L},
$$

where $L$ is the total length of the FP resonator. This fluctuating force will cause fluctuations in the positions of the mirrors, thus causing fluctuations in the spatial shift of the optical field with respect to the mirror $B$ and the nonlinear slabs attached to it:

$$
\delta \widetilde{x}(\Omega)=-\frac{3}{2} \frac{\delta \widetilde{F}(\Omega)}{m \Omega^{2}}=-\frac{3}{2} K \frac{\omega_{\mathrm{opt}}}{\omega_{e}} \frac{\hbar}{L} \delta \widetilde{N}_{e}(\Omega),
$$

where $\delta \widetilde{x}(\Omega), \delta \widetilde{F}(\Omega)$, and $\delta \widetilde{N}_{e}(\Omega)$ are Fourier components of the corresponding quantities, $m$ is the mass of each of the mirrors, and the factor $\frac{3}{2}$ comes about when motion of all three mirrors is taken into account.

Now we are ready to describe our QND method, but first the following simple remark must be made. Suppose for a moment that all of the mirrors are rigidly fixed. As already mentioned above, fluctuations in $N_{e}$, by changing the optical coefficient of refraction of the slabs [Eq. (16)], will redistribute optical energy between the left and right parts of the FP resonator. A straightforward calculation [Eq. (A16) in Appendix A] shows that this alone will change the optical field inside the nonlinear slabs so that

$$
\delta E_{1}=-\delta E_{2}=E_{0} \frac{n \omega_{\mathrm{opt}} l}{c \sqrt{2}} \delta n
$$

thereby simulating a spatial shift of the optical field as in Eq. (6). Here $l$ is the width of the nonlinear slab, $n \equiv n_{1}=n_{2}$, $\delta n \equiv \delta n_{1}=-\delta n_{2}$, and $E_{0}$ is the peak amplitude of the optical standing wave inside the FP resonator.

Now if we release the mirrors, the back action (18) will affect our reading as well, and the total fluctuations of the optical field inside the nonlinear slabs will be given by

$$
\delta \widetilde{E}_{1}(\Omega)-\delta \widetilde{E}_{2}(\Omega)=\sqrt{2} n E_{0} \frac{\omega_{\mathrm{opt}} l}{c}\left(1-6 \frac{\omega_{\mathrm{opt}}^{2}}{\Omega^{2}} \frac{\hbar N}{m c^{2} \tau}\right) \delta n(\Omega),
$$


where $\tau=L / c$; cf. Eq. (A17) of Appendix A. From the above equation we see that for a given frequency $\Omega=\Omega_{0}$ we can adjust $N$ in such a way that $\delta \widetilde{E}_{1}(\Omega)=\delta \widetilde{E}_{2}(\Omega)=0$ and thus the readout system does not register any fluctuations due to the back action (but only for that value of $\Omega$ ). Thus a QND measurement is performed. The relationship between the QND angular frequency $\Omega_{0}$ and the number $N$ of optical photons in the Fabry-Perot resonator is

$$
\Omega_{0}=\sqrt{\frac{6 \hbar N}{m c L}} \omega_{\mathrm{opt}},
$$

see Eq. (3) of Sec. I.

The essential reason that this readout is QND is that it registers not only the fluctuations of the mirrors' displacement $x$ due to back action [the second term in large parentheses in Eq. (17)], but also directly the back action force (the first term). Thus a position-momentum correlation is introduced into the measurement procedure, and such correlations are known to make QND possible [4]. For $L=4 \mathrm{~km}$, $\Omega / 2 \pi=60 \mathrm{~Hz}, \omega_{\mathrm{opt}}=3 \times 10^{15} \mathrm{~s}^{-1}$, and $m=10 \mathrm{~kg}$ the necessary number of optical photons to perform QND is

$$
N=\frac{1}{6} \frac{\Omega_{0}^{2}}{\omega_{\mathrm{opt}}^{2}} \frac{m c^{2} \tau}{\hbar} \sim 2.8 \times 10^{20} .
$$

The QND measurement described above is clearly narrowband. In principle one can dynamically tune the frequency at which the QND is performed by changing the laser power and thus changing the number of optical photons $N$ in the resonator, provided that the frequency of the signal changes slowly compared to the ring-down rate of the optical resonator. In practice the issues of fluctuations in $N$ and stability of control systems may be a serious obstacle for such dynamical tuning. Analysis of these and other practical difficulties is beyond the scope of the present work.

Appendix B considers a particular scheme for measuring of the phase of the microwave oscillator. In this scheme the oscillator is coupled to a transmission line, and the physically measured quantity is the phase quadrature of the outgoing electromagnetic wave propagating along the transmission line. Having specified fully the measurement model, we find that if the bandwidth of measurement is $\Delta \Omega$ then the signal-to-noise ratio for the narrow-band QND measurement can be as high as

$$
\begin{aligned}
\left(\frac{S}{N}\right)_{\mathrm{QND}}^{2} & \sim\left(\frac{S}{N}\right)_{\mathrm{SQL}}^{2} \frac{\Omega_{0}}{\Delta \Omega} \\
& =\frac{1}{2 \pi} \frac{\Omega_{0}}{\Delta \Omega} \int_{\Omega_{0}-\Delta \Omega}^{\Omega_{0}+\Delta \Omega} \frac{m \Omega^{2} L^{2}|h(\widetilde{\Omega})|^{2}}{\hbar} d \Omega .
\end{aligned}
$$

The above signal-to-noise ratio is achieved when the pumping power of the microwave oscillator is given by

$$
W_{\text {optimal }}=\frac{V^{2}}{32 \pi^{2} \chi^{(3) 2} N \tau}\left(\frac{\Omega_{0}}{\omega_{\text {opt }}}\right)^{2}\left(\frac{L}{l}\right)^{2} \frac{1}{\hbar \omega_{e}} \frac{\Omega_{0}}{\Delta \Omega}=W_{\mathrm{SQL}} \frac{\Omega_{0}}{\Delta \Omega},
$$

where $V$ is the volume of the capacitor and $W_{\text {SQL }}$ is the minimal power necessary to achieve the SQL sensitivity level; cf. Eq. (B13) of Appendix B and Eq. (4) of the Introduction. For $V=(0.01 \mathrm{~mm})^{3}, \omega_{e}=10^{11} \mathrm{~s}^{-1}$, and for other parameters having numerical values as in Eq. (22), we get $W_{\text {opt }}=0.1 \mathrm{~W}\left(\Omega_{0} / \Delta \Omega\right.$ ) (cf. the $1 \mathrm{~kW}$ of the optical power required to achieve the SQL in a conventional interferometric scheme).

While we have not devised a general proof, it seems likely that no other microwave readout scheme can operate with a power less than in Eq. (24); expression (24) is probably a general relation for optimally designed microwave readout schemes.

\section{HIGHER ORDER OPTICAL EFFECTS: FUNDAMENTAL SENSITIVITY LIMIT}

In this section we identify and discuss a fundamental limit on the sensitivity of the BK readout system-a limit that applies whether or not the system is being operated in a QND mode.

In an interferometric GW detector, mirrors are installed on the surfaces of test masses, which have internal elastic mechanical modes of frequencies $\Omega_{m} / 2 \pi \geqslant 12 \mathrm{kHz}$. The noise curve of the interferometer will have large peaks near these frequencies. When photons of frequency $\omega_{\text {opt }}$ interact with walls oscillating with the frequancy $\Omega_{m}$, some of the photons will be up or down converted to frequencies $\omega_{\text {opt }} \pm \Omega_{m}$. These up or down converted photons in turn interact with the "noisy" walls, and if there is a nonzero component of the mirrors' motion at $\Omega_{m} \pm \Omega$, then some of the photons will up or down convert a second time to frequencies $\omega_{0} \pm \Omega$. If $\Omega$ is the frequency of detection, then this second-order process of double frequency conversion will be registered by the BK readout system as a signal from a gravitational wave.

The perturbation theory for FP resonators with moving walls is worked out in detail in Appendix C. Here just the main result is quoted. From Eq. (C26) the noise curve in units of $1 / \sqrt{\mathrm{Hz}}$ is given by

$$
\sqrt{S_{h}(\Omega)} \sim \frac{1}{3 \pi} \frac{\omega_{\mathrm{opt}} k_{B} T_{e} \sqrt{\gamma_{m}}}{m L \Omega_{m}^{2} \Omega^{2} \tau c} \sqrt{\Omega \tau+\epsilon},
$$

where $T_{e}$ is the temperature of the test masses, $\gamma_{m}$ is the damping rate of their mechanical modes, and $\epsilon \sim \max \left(\delta \chi^{(3)} / \chi^{(3)}, \delta / / \lambda\right)$. Here $\delta \chi \equiv \chi_{1}^{(3)}+\chi_{2}^{(3)}, \chi^{(3)} \equiv \chi_{1}^{(3)}, \lambda$ is the wavelength of light in the resonator and $\delta l$ is the spatial offset of the slabs from the position in shown Fig. 2. For $L=4 \mathrm{~km}, \Omega=60 \mathrm{rad} / \mathrm{s}, T_{e}=300 \mathrm{~K}, \Omega_{m}=7.2 \times 10^{4} \mathrm{rad} / \mathrm{s}$, $m=10 \mathrm{~kg}$, and $\gamma_{m}=10^{-8} \Omega_{m}$ we get the noise level of

$$
\sqrt{S_{h}} \sim 10^{-28} \sqrt{(\Omega \tau+\epsilon)} / \sqrt{\mathrm{Hz}} .
$$

It is not unimaginable that future interferometers will achieve sensitivities $\sqrt{S_{h}} \sim 10^{-29} / \sqrt{\mathrm{Hz}}$ for low frequency $(10-100 \mathrm{~Hz})$ narrow-band signals (by, e.g., using the QND technique described in this paper). In this case, Eqs. (25) and (26) show that higher order effects will give rise to a "fundamental" low frequency noise limit of magnitude 


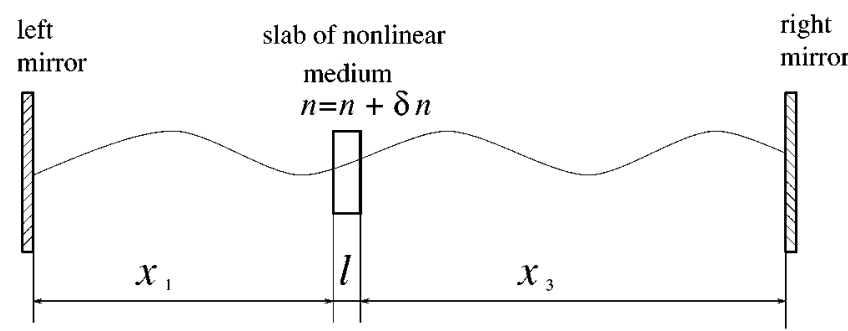

FIG. 3. The changing coefficient of refraction of the slab of nonlinear medium will redistribute optical energy between the left and right parts of the Fabry-Perot resonator.

$$
\sqrt{S_{h}(\Omega)} \sim 10^{-28}\left(\frac{60 \mathrm{rad} / \mathrm{s}}{\Omega}\right)^{2} / \sqrt{\mathrm{Hz}} .
$$

\section{CONCLUSIONS}

In this paper we have shown that a practical QND measurement might be possible for a narrow-band measurement by a gravitational wave interferometer using a BK readout system. Also it was shown that second-order effects set a "fundamental" limit on the precision of the measurement.

\section{ACKNOWLEDGMENTS}

I want to thank Vladimir Braginsky for suggesting the problem, for interesting and useful discussions, Kip Thorne for reading carefully over the manuscript and making many useful suggestions, and Farid Khalili and Sergei Vyatchanin for interesting discussions. This work was supported in part by NSF Grant No. PHY-9424337.

\section{APPENDIX A: PHYSICS OF THE NONLINEAR MEDIUM INSIDE THE BK FABRY-PEROT RESONATOR}

First consider one slab of nonlinear medium positioned inside a FP resonator. Let $x_{1}$ (Fig. 3) be the total path length from the left mirror to the left edge of the slab, $x_{3}$ be the path length from the right mirror to the right edge of the slab, and $l$ be the width of the slab. For simplicity of the calculation we assume $l \ll \lambda$ where $\lambda$ is the wavelength of light in the resonator. Also for convenience define $\tau_{1}=x_{1} / c, \tau_{2}=l / c$, $\tau_{3}=x_{3} / c$.

The eigenfrequencies $\omega$ of this optical resonator were worked out in [3]. They satisfy the following eigenequation:

$$
\begin{aligned}
\sin (\omega \tau)= & (n-1) \sin \left(n \omega \tau_{2}\right)\left[\sin \left(\omega \tau_{1}\right) \sin \left(\omega \tau_{3}\right)\right. \\
& \left.+\frac{1}{n} \cos \left(\omega \tau_{1}\right) \cos \left(\omega \tau_{3}\right)\right]
\end{aligned}
$$

where $\tau=\tau_{1}+\tau_{3}+n \tau_{2}$. This equation has approximate solutions

$$
\omega=\omega_{0}+\left(n^{2}-1\right) \omega_{0} \frac{\tau_{2}}{2 \tau}\left\{\cos \left[\omega_{0}\left(\tau_{1}-\tau_{3}\right)\right]-1\right\},
$$

where $\omega_{0}=\pi k / \tau$, and $k$ is any integer. When the slab's index of refraction $n$ changes, $\omega$ changes accordingly:

$$
\frac{d \omega}{d n}=\frac{n \omega^{0} \tau_{2}}{\tau}\left\{\cos \left[\omega^{0}\left(\tau_{1}-\tau_{3}\right)\right]-1\right\}
$$

The total optical energy contained in the resonator is

$$
U=N \hbar \omega,
$$

where $N$ is the number of optical photons. We can find all of the forces acting on the mirrors by taking derivatives of $U$ with respect to $\tau_{1}$ and $\tau_{3}$. For example,

$$
F_{\text {left }}=-\frac{N \hbar}{c} \frac{\partial \omega}{\partial \tau_{1}}
$$

and

$$
F_{\text {right }}=-\frac{N \hbar}{c} \frac{\partial \omega}{\partial \tau_{3}}
$$

where $F_{\text {left }}$ and $F_{\text {right }}$ are the forces acting on the left and the right mirrors, respectively, with the positive direction being out of the resonator. When taking derivatives of $\omega$ one has to keep in mind that $\omega_{0}$ also depends on $\tau_{1}$ and $\tau_{3}$.

The force acting on the slab of nonlinear medium is $F_{\text {left }}-F_{\text {right }}$. The total spatial shift of the optical wave with respect to the slab due to the forces acting on the end mirrors and the slab itself is

$$
\delta \widetilde{x}(\Omega)=-\frac{3}{2 m \Omega^{2}}\left[\widetilde{F}_{\text {left }}(\Omega)-\widetilde{F}_{\text {right }}(\Omega)\right],
$$

where "tildas" stand for Fourier transforms. If $F_{\text {left }}$ and $F_{\text {right }}$ are produced by a fluctuating index of refraction $n=n_{0}+\delta n$ then on substituting Eqs. (A5) and (A6) into Eq. (A7) we get

$$
\delta \widetilde{x}(\Omega)=\frac{3 N \hbar}{2 m \Omega^{2}}\left(\frac{\partial}{\partial \tau_{1}}-\frac{\partial}{\partial \tau_{3}}\right) \frac{d \omega}{d n} \delta \tilde{n}(\Omega) .
$$

By then putting Eqs. (A3) and (A8) together we obtain

$$
\delta \widetilde{x}(\Omega)=-\frac{3 N \hbar n}{m c} \frac{\omega^{2}}{\Omega^{2}} \frac{\tau_{2}}{\tau} \sin \left[\omega\left(\tau_{1}-\tau_{3}\right)\right] \delta \widetilde{n}(\Omega) .
$$

For the two slabs of opposite nonlinearities $\left(\delta n_{1}=-\delta n_{2}=\delta n\right)$ in the configuration of Fig. 2 their two contributions add up to give

$$
\delta \tilde{x}(\Omega)=-6 \frac{N n \hbar}{m c} \frac{\omega^{2}}{\Omega^{2}} \frac{\tau_{2}}{\tau} \delta \tilde{n}(\Omega) .
$$

The above expression is a manifestation of the back action as explained in Sec. II.

Now let the amplitude of the optical electric field in the left part of the resonator be

$$
E_{\text {left }}=E_{0} \sin \left(\frac{\omega x}{c}\right),
$$

where $x$ is the spatial coordinate with the origin at the left wall. Then the field in the middle of the left slab is given by 


$$
E_{1}=E_{0}\left[\sin \left(\omega \tau_{1}\right)+\frac{1}{n} \cos \left(\omega \tau_{1}\right) \sin \left(\frac{n \omega \tau_{2}}{2}\right)\right]+O\left[\left(\omega \tau_{2}\right)^{2}\right]
$$

Now

$$
\frac{d E_{1}}{d n} \simeq \frac{d E_{0}}{d n} \sin \left(\omega \tau_{1}\right)+E_{0} \tau_{1} \cos \left(\omega \tau_{1}\right) \frac{d \omega}{d n} .
$$

In the case when two slabs are present inside the FP resonator, their contributions to the frequency and field changes add up linearly (since the perturbations are very small). For the configuration of Fig. 2 we see from Eq. (A3) that $d \omega / d n=0$, so

$$
\frac{d E_{1}}{d n}=\frac{d E_{0}}{d n} \sin \left(\omega \tau_{1}\right)
$$

But $F_{\text {left }} \propto E_{0}^{2}$, so

$$
\frac{d E_{0} / d n}{E_{0}}=\frac{1}{2} \frac{d F_{\text {left }} / d n}{F_{\text {left }}}=-\frac{\tau}{\omega} \frac{d}{d n} \frac{\partial \omega}{\partial \tau_{1}} .
$$

Putting Eq. (A3) and Eq. (A14) into Eq. (A15) and doing exactly the same calculation for the second slab, we obtain

$$
\delta E_{1}=-\delta E_{2}=E_{0} \frac{n \omega_{\mathrm{opt}} l}{\sqrt{2} c} \delta n
$$

for the case when mirror $B$ is in the middle of the resonator. Combining this with the back action from Eq. (A10) we finally get Eq. (20) of Sec. III:

$$
\delta \widetilde{E}_{1}(\Omega)-\delta \widetilde{E}_{2}(\Omega)=\sqrt{2} n E_{0} \frac{\omega_{\text {opl }} l}{c}\left(1-6 \frac{\omega_{\mathrm{opt}}^{2}}{\Omega^{2}} \frac{\hbar N}{m c^{2} \tau}\right) \delta n(\Omega) .
$$

\section{APPENDIX B: CALCULATION OF OPTIMAL MICROWAVE POWER AND SIGNAL-TO-NOISE RATIO FOR A QND MEASUREMENT}

Consider the microwave oscillator as shown in Fig. 2. In order to get information about the phase of the oscillator, we have to couple it to the outside world. Whatever the nature of this coupling is, it will cause dissipation of the induced oscillations and hence, by the fluctuation-dissipation theorem, give birth to a fluctuating component of the oscillator's current.

For concreteness, we model this coupling by an open transmission line of impedance $R$. We assume that the oscillator, consisting of the capacitor $C$ and inductor $\mathcal{L}$, is driven on resonance by a generator $G$ with a voltage output of amplitude $V_{0}$ (see Fig. 2). We also assume the transmission line encompasses all of the dissipation present in the oscillator, i.e., more generally, that we can access all of the information escaping from the oscillator. And finally, we set the tempera- ture of the outside world to 0 (in reality, one will have to cool the oscillator to temperatures below the ones corresponding to a microwave frequency). The ingoing vacuum modes drive fluctuations in the circuit as described above, and the phase of the outgoing wave contains information about the phase of the oscillator.

The ingoing modes are described by the positive frequency part of a voltage operator

$$
V_{\mathrm{in}}=\int_{0}^{\infty} d \omega \sqrt{R \hbar \omega} a_{\mathrm{in}}(\omega) e^{-\imath \omega t},
$$

where $a_{\text {in }}(\omega)$ is the annihilation operator for the ingoing mode of frequency $\omega$ normalized so that $\left\langle 0\left|a_{\text {in }}(\omega) a_{\text {in }}^{\dagger}\left(\omega^{\prime}\right)\right| 0\right\rangle=\delta\left(\omega-\omega^{\prime}\right)$. Then the Fourier component of the outgoing wave is

$$
V_{\text {out }}\left(\omega_{e}+\Omega\right)=\sqrt{R \hbar \omega_{e}} \frac{\alpha+{ }_{\imath} \Omega}{\alpha-\imath \Omega} a_{\text {in }}\left(\omega_{e}+\Omega\right)+\frac{V_{0} \delta \omega_{e}(\Omega)}{2 \Omega+\imath \alpha},
$$

where $\alpha=R / \mathcal{L}$ is the ringdown rate of the microwave oscillator and $\delta \omega_{e}$ is the variation in the oscillator's resonant frequency due to fluctuating optical fields in the slabs of nonlinear medium, as explained in Sec. II:

$$
\delta \omega_{e}=\frac{8 \pi^{2} \chi^{(3)} l N \hbar \omega_{\mathrm{opt}} \omega_{e}}{\sqrt{2} V L} \frac{\delta E_{1}-\delta E_{2}}{E_{0}} .
$$

Here $V$ is the volume of the capacitor. The change of the optical field inside the slabs is given by

$$
\begin{aligned}
\delta E_{1}(\Omega)-\delta E_{2}(\Omega)= & \sqrt{2} E_{0} n \omega_{\mathrm{opt}} \tau_{2}\left(1-6 \frac{N \hbar}{m c^{2} \tau} \frac{\omega_{\mathrm{opt}}^{2}}{\Omega^{2}}\right) \delta n(\Omega) \\
& +\sqrt{2} E_{0} \frac{\omega_{\mathrm{opt}}}{c} x_{s}(\Omega)
\end{aligned}
$$

where the first term on the right-hand side is due to the fluctuating index of refraction of the slabs [cf. Eq. (20) of Sec. III and discussion therein], and the second term is due to the GW-induced relative displacement $x_{s}$ of the slabs with respect to the standing optical wave. The fluctuations $\delta n$ of the indices of refraction of the nonlinear slabs in the above expression are caused by the voltage fluctuations on the plates of the capacitor, which in turn can be traced to the incoming vacuum modes of the transmission line:

$$
\begin{aligned}
\delta n(\Omega)= & -l \frac{2 \pi \chi^{(3)} \sqrt{R \hbar \omega_{e}} V_{0} \omega_{e}^{2}}{\alpha(2 \Omega+\imath \alpha) d^{2}}\left[a_{\mathrm{in}}\left(\omega_{e}+\Omega\right)\right. \\
& \left.+a_{\mathrm{in}}^{\dagger}\left(\omega_{e}-\Omega\right)\right],
\end{aligned}
$$

where $d$ is the distance between the plates of the capacitor. Collecting Eqs. (B2), (B3), (B4), and (B5) together, we can write down the expression for the phase quadrature of the outgoing wave in the transmission line, which is the measured readout signal: 


$$
\begin{aligned}
r(\Omega)= & {\left[V_{\text {out }}\left(\omega_{e}+\Omega\right)-V_{\mathrm{out}}^{\dagger}\left(\omega_{e}-\Omega\right)\right] / V_{0}=\frac{\sqrt{R \hbar \omega_{e}}}{V_{0}} \frac{\alpha+\imath \Omega}{\alpha-\imath \Omega}\left[a_{\mathrm{in}}\left(\omega_{e}+\Omega\right)-a_{\mathrm{in}}^{\dagger}\left(\omega_{e}-\Omega\right)\right]+\frac{8 \pi^{2} V_{0} \chi^{(3)} l N \hbar \omega_{\mathrm{opt}}^{2} \omega_{e}}{V_{0}(2 \Omega+\imath \alpha) V L c} } \\
& \times\left\{x_{s}-\frac{8 \pi^{2} \chi^{(3)} V_{0} \sqrt{R \hbar \omega_{e}} l}{R V(2 \Omega+\imath \alpha)}\left(1-\frac{6 N \hbar}{m c^{2} \tau} \frac{\omega_{\mathrm{opt}}^{2}}{\Omega^{2}}\right)\left[a_{\mathrm{in}}\left(\omega_{e}+\Omega\right)+a_{\mathrm{in}}^{\dagger}\left(\omega_{e}-\Omega\right)\right]\right\} .
\end{aligned}
$$

The measured $x$ is then given by

$$
\begin{aligned}
x_{\text {measured }}(\Omega)= & x_{s}(\Omega)-\frac{8 \pi^{2} \chi^{(3)} \sqrt{\hbar \omega_{e} W} l}{V(2 \Omega+\imath \alpha)}\left(1-\frac{6 N \hbar}{m c^{2} \tau} \frac{\omega_{\mathrm{opt}}^{2}}{\Omega^{2}}\right)\left[a_{\mathrm{in}}\left(\omega_{e}+\Omega\right)+a_{\mathrm{in}}^{\dagger}\left(\omega_{e}-\Omega\right)\right]+\frac{V L c(2 \Omega+\imath \alpha) \sqrt{\hbar \omega_{e}}}{16 \pi^{2} \chi^{(3)} l N \hbar \omega_{\mathrm{opt}}^{2} \omega_{e} \sqrt{W}} \\
& \times\left[a_{\mathrm{in}}\left(\omega_{e}+\Omega\right)-a_{\mathrm{in}}^{\dagger}\left(\omega_{e}-\Omega\right)\right],
\end{aligned}
$$

where $W=V_{0}^{2} / R$ is the power pumped into the microwave oscillator by the generator $G$. The corresponding spectral density of the Gaussian noise seen by the readout system is

$$
S_{x}(\Omega)=\left(\frac{8 \pi^{2} \chi^{(3)} l}{V}\right)^{2} \frac{\hbar \omega_{e} W}{4 \Omega^{2}+\alpha^{2}}\left(1-\frac{6 N \hbar}{m c^{2} \tau} \frac{\omega_{\mathrm{opt}}^{2}}{\Omega^{2}}\right)^{2}+\left(\frac{V L c}{16 \pi^{2} \chi^{(3)} l N \hbar \omega_{\mathrm{opt}}^{2} \omega_{e}}\right)^{2} \frac{\left(4 \Omega^{2}+\alpha^{2}\right) \hbar \omega_{e}}{W} .
$$

The first term on the right-hand side corresponds to the back action noise and the second term corresponds to the intrinsic noise of the measuring device.

We aim to perform a measurement with a narrow frequency band centered around the frequency $\Omega_{0}$ at which the back action noise is zero:

$$
\Omega_{0}=\sqrt{\frac{6 N \hbar}{m \tau}} \frac{\omega_{\mathrm{opt}}}{c} .
$$

We write $S_{x}$ as a Taylor expansion in frequency around $\Omega_{0}$.

$$
S_{x}(\Omega) \simeq A\left(\Omega_{0}\right) W \frac{\left(\Omega-\Omega_{0}\right)^{2}}{\Omega_{0}^{2}}+\frac{B\left(\Omega_{0}\right)}{W},
$$

where $A$ and $B$ can be read from Eq. (B8). If the relevant bandwidth is $\Delta \Omega$ then we place a limit $\left(\Omega-\Omega_{0}\right)^{2} \leqslant \Delta \Omega^{2}$ and

$$
S_{x}(\Omega) \leqslant A\left(\Omega_{0}\right) W \frac{\Delta \Omega^{2}}{\Omega_{0}^{2}}+\frac{B\left(\Omega_{0}\right)}{W} .
$$

Minimizing the right-hand side of the above equation with respect to $W$, we find the expression for the minimum noise in a fixed bandwidth:

$$
S_{\text {xoptimal }}(\Omega) \leqslant 2 \sqrt{A B} \frac{\Delta \Omega}{\Omega_{0}}=\frac{1}{2 \pi^{2}} \frac{\lambda^{2} \tau}{N} \frac{\Delta \Omega}{\Omega_{0}} \sim S_{x \mathrm{SQL}}\left(\Omega_{0}\right) \frac{\Delta \Omega}{\Omega_{0}},
$$

which is achieved at the input power

$$
W_{\text {optimal }}=\frac{V^{2}}{32 \pi^{2} \chi^{(3) 2} N \tau}\left(\frac{\Omega_{0}}{\omega_{\text {opt }}}\right)^{2}\left(\frac{L}{l}\right)^{2} \frac{1}{\hbar \omega_{e}} \frac{\Omega_{0}}{\Delta \Omega} .
$$

In the above expressions $\lambda$ is the wavelength of light inside the FP resonator and $S_{\mathrm{SQL}}\left(\Omega_{0}\right)$ is the standard quantum limit noise at the frequency $\Omega_{0}$ for a free mass. Clearly, the signal-to-noise ratio for this narrow-band measurement is $\sqrt{\Omega_{0} / \Delta \Omega}$ greater than that in the case of the SQL:

$$
\left(\frac{S}{N}\right) \sim\left(\frac{S}{N}\right)_{\mathrm{SQL}} \sqrt{\frac{\Omega_{0}}{\Delta \Omega}}
$$

\section{APPENDIX C: PERTURBATION THEORY FOR FABRY-PEROT CAVITY WITH MOVING WALLS}

In this appendix we derive a formal series for the optical field inside a Fabry-Perot resonator which is pumped by a monochromatic laser beam and the walls of which are free to perform motions small compared to the wavelength of light $\lambda$. The expansion parameter is $\delta x / \lambda$, where $\delta x$ is the change of length of the resonator. For our purposes we are only interested in expanding up to $(\delta x / \lambda)^{2}$; and we use this formal series to derive Eq. (25).

The following situation is considered: for simplicity we assume that light is pumped on resonance by a laser beam $E_{\text {in }}=\alpha e^{-l\left(\omega_{\text {opt }} t-k x\right)}$ through the left mirror which is at rest and has reflectivity $r$ and transmissivity $T$. For concreteness it is assumed that the fluctuations in length $\delta x$ originate from the motion of the right mirror which is assumed to be perfectly reflecting. Further, we assume that the plain wave approximation is applicable and hence the optical field inside the resonator satisfies the one-dimensional wave equation:

$$
\left(\frac{\partial^{2}}{\partial t^{2}}-c^{2} \frac{\partial^{2}}{\partial x^{2}}\right) A(x, t)=0 .
$$

The general solution of of the above equation is

$$
A(x, t)=f\left(t+\frac{x}{c}\right)+g\left(t-\frac{x}{c}\right),
$$

where $f$ and $g$ are arbitrary functions. The boundary conditions at the left mirror $(x=0)$ and at the right mirror $(x=L+\delta x)$ read, respectively, 


$$
g(t)-r f(t)=T \alpha e^{-\imath \omega_{\mathrm{opt}}}
$$

and

$$
f\left(t+\tau_{0}+\frac{\delta x}{c}\right)+g\left(t-\tau_{0}-\frac{\delta x}{c}\right)=0
$$

where $\tau_{0}=L / c$. Eliminating $g(t)$ from these two equations, we get

$$
\begin{array}{r}
f\left(t+\tau_{0}+\frac{\delta x}{c}\right)-r f\left(t-\tau_{0}-\frac{\delta x}{c}\right) \\
=T \alpha e^{-\imath \omega_{\mathrm{opt}} t} e^{\imath \omega_{\mathrm{opt}} \tau_{0}} e^{\imath\left(\omega_{\mathrm{opt}} / c\right) \delta x}
\end{array}
$$

or, expanding in $\delta x$ up to second order,

$$
\begin{aligned}
f\left(t+\tau_{0}\right)-r f\left(t-\tau_{0}\right) & \\
= & T \alpha e^{-\imath \omega_{\mathrm{opt}} t} e^{\imath \omega_{\mathrm{opt}} \tau_{0}}\left(1+l \frac{\omega_{\mathrm{opt}}}{c} \delta x-\frac{\omega_{\mathrm{opt}}^{2}}{c^{2}} \delta x^{2}\right) \\
& -\frac{1}{c}\left[f^{\prime}\left(t+\tau_{0}\right)+r f^{\prime}\left(t-\tau_{0}\right)\right] \delta x \\
& -\frac{1}{2 c^{2}}\left[f^{\prime \prime}\left(t+\tau_{0}\right)-r f^{\prime \prime}\left(t-\tau_{0}\right)\right] \delta x^{2} .
\end{aligned}
$$

We take a Fourier tranform of the above equation and then solve it by iterations:

$$
f(\omega)=f^{(0)}(\omega)+f^{(1)}(\omega)+f^{(2)}(\omega)+\cdots,
$$

where

$$
f^{(0)}(\omega)=\frac{T \alpha}{1-r} \delta\left(\omega-\omega_{\mathrm{opt}}\right)
$$

$$
f^{(1)}(\omega)=\frac{2 \imath \omega_{\mathrm{opt}} T \alpha}{c\left[(1-r) \cos \left(\omega \tau_{0}\right)-\imath(1+r) \sin \left(\omega \tau_{0}\right)\right](1-r)}
$$

$$
\times \delta x\left(\omega-\omega_{\text {opt }}\right)
$$

$$
\begin{aligned}
f^{(2)}(\omega)= & -\frac{4 \omega_{\mathrm{opt}}^{2} T \alpha}{c^{2}\left[(1-r) \cos \left(\omega \tau_{0}\right)-{ }_{l}(1+r) \sin \left(\omega \tau_{0}\right)\right](1-r)} \\
& \times \int d \omega^{\prime} \frac{\cos \left(\omega^{\prime} \tau_{0}\right) \delta x\left(\omega^{\prime}-\omega_{\mathrm{opt}}\right) \delta x\left(\omega-\omega^{\prime}\right)}{(1-r) \cos \left(\omega^{\prime} \tau_{0}\right)-\imath(1+r) \sin \left(\omega^{\prime} \tau_{0}\right)} .
\end{aligned}
$$

When writing down the above terms we took into account the fact that $1-r \ll 1$. The structure of $f^{(2)}$ is clear: it corresponds to upconversion of light at frequency $\omega_{\text {opt }}$ to an intermediate frequency $\omega^{\prime}$ and then from $\omega^{\prime}$ to $\omega$, with $\omega^{\prime}$ being integrated over. From Eqs. (C2) and (C3),

$$
A(x, \omega) \simeq-2 \imath \sin \left(\frac{\omega}{c} x\right) f(\omega)
$$

The BK readout system detects the square of the amplitude of the optical field:

$$
S(x, t) \equiv|A(x, t)|^{2}=S^{(0)}(x, t)+S^{(1)}(x, t)+S^{(2)}(x, t)+\cdots,
$$

where

$$
\begin{gathered}
S^{(0)}(x, \Omega)=4 \sin ^{2}\left(\frac{\omega_{\mathrm{opt}}}{c} x\right) C^{2} \delta(\Omega), \\
S^{(1)}(x, \Omega)=0
\end{gathered}
$$

and

$$
\begin{aligned}
S^{(2)}(x, \Omega)= & -\left(\frac{2 \omega_{\mathrm{opt}} C}{c}\right)^{2} \int\left\{\frac{\sin \left\{\left[\left(\omega_{\mathrm{opt}}-\Omega^{\prime}\right) / c\right] x\right\} \sin \left\{\left[\left(\omega_{\mathrm{opt}}+\Omega-\Omega^{\prime}\right) / c\right] x\right\}}{\mathcal{L}\left(\Omega^{\prime}\right) \mathcal{L}\left(\Omega-\Omega^{\prime}\right)}\right. \\
& \left.+\frac{2\left(\sin \left\{\left[\left(\omega_{\mathrm{opt}}+\Omega\right) / c\right] x\right\}+\sin \left\{\left[\left(\omega_{\mathrm{opt}}-\Omega\right) / c\right] x\right\}\right)}{\mathcal{L}(\Omega) \mathcal{L}\left(\Omega^{\prime}\right)}\right\} \delta x\left(\Omega^{\prime}\right) \delta x\left(\Omega-\Omega^{\prime}\right) d \Omega^{\prime} .
\end{aligned}
$$

In the above expression $C \equiv T \alpha /(1-r)$ and $\mathcal{L}(\Omega)$ $\equiv(1-r) \cos (\Omega \tau)+(1+r) \sin (\Omega \tau)$.

In real interferometers $\delta x$ represents, for example, motion of the surface of the mirror due to the thermal excitation of the test mass' internal modes. In what follows the contribution from the internal mode of lowest frequency is considered and then it will be shown that the sum of contributions of all the higher modes will have the same order of magnitude. It is assumed that the thermal noise is a Markoff Gaussian process, and therefore is described by the following equation:

$$
\delta x(\Omega)=\frac{F(\Omega)}{\Omega^{2}-\Omega_{m}^{2}+\imath \gamma_{m} \Omega},
$$

where $\Omega_{m}$ is the eigenfrequency of the mechanical mode, $\gamma_{m}$ is the damping rate, and $F(\Omega)$ is the Langevin force satisfying

$$
\left\langle F\left(\Omega_{1}\right) F\left(\Omega_{2}\right)\right\rangle=\frac{D}{2 \pi} \delta\left(\Omega_{1}+\Omega_{2}\right),
$$


where $D=k_{B} T_{e} \gamma_{m} / m^{*}$ is the velocity diffusion rate. Here $m^{*}$ is the effective mass of the mode (approximately given by the mirror mass $m$ ), $k_{B}$ is Boltzmann's constant, and $T_{e}$ is the temperature of the enviroment. To calculate the spectral density of the fluctuations of $S^{(2)}$ (the goal of this analysis) we will need the four-point correlation function:

$$
\begin{aligned}
\left\langle F\left(\Omega_{1}\right)\right. & \left.F\left(\Omega_{2}\right) F\left(\Omega_{3}\right) F\left(\Omega_{4}\right)\right\rangle \\
= & \frac{D^{2}}{8 \pi^{2}}\left[\delta\left(\Omega_{1}+\Omega_{2}\right) \delta\left(\Omega_{3}+\Omega_{4}\right)+\delta\left(\Omega_{1}+\Omega_{3}\right)\right. \\
& \left.\times \delta\left(\Omega_{2}+\Omega_{4}\right)+\delta\left(\Omega_{1}+\Omega_{4}\right) \delta\left(\Omega_{2}+\Omega_{3}\right)\right] .
\end{aligned}
$$

Using the above expression, Eq. (C15) and Eq. (C16), we obtain

$$
\begin{aligned}
\left\langle S^{(2)}\left(x, \Omega_{1}\right) S^{(2)}\left(x, \Omega_{2}\right)\right\rangle= & \left(2 \omega_{0} C\right)^{4}\left[M_{1} \delta\left(\Omega_{1}\right) \delta\left(\Omega_{2}\right)\right. \\
& \left.+M_{2}\left(x, \Omega_{1}\right) \delta\left(\Omega_{1}+\Omega_{2}\right)\right] .
\end{aligned}
$$

Here $M_{2}(\Omega)$, which characterizes the spectral density of fluctuations of $S^{(2)}$, is given by

$$
\begin{aligned}
M_{2}(x, \Omega)= & \frac{D^{2}}{8 \pi^{2}} \int\left[K\left(\Omega, \Omega^{\prime}\right) K\left(-\Omega,-\Omega^{\prime}\right)+K\left(\Omega, \Omega^{\prime}\right)\right. \\
& \left.\times K\left(-\Omega,-\Omega+\Omega^{\prime}\right)\right] d \Omega^{\prime},
\end{aligned}
$$

where

$$
\begin{aligned}
K\left(\Omega, \Omega^{\prime}\right)= & \frac{1}{\mathcal{L}\left(\Omega^{\prime}\right)\left(\Omega^{\prime 2}-\Omega_{m}^{2}+\imath \gamma_{m} \Omega^{\prime}\right)\left[\left(\Omega-\Omega^{\prime}\right)^{2}-\Omega_{m}^{2}+\imath \gamma_{m}\left(\Omega-\Omega^{\prime}\right)\right]} \\
& \times\left\{\frac{\sin \left\{\left[\left(\omega_{\mathrm{opt}}-\Omega^{\prime}\right) / c\right] x\right\} \sin \left\{\left[\left(\omega_{\mathrm{opt}}+\Omega-\Omega^{\prime}\right) / c\right] x\right\}}{\mathcal{L}\left(\Omega-\Omega^{\prime}\right)}\right. \\
& \left.+\frac{\left(\sin \left\{\left[\left(\omega_{\mathrm{opt}}+\Omega\right) / c\right] x\right\}+\sin \left\{\left[\left(\omega_{\mathrm{opt}}-\Omega\right) / c\right] x\right\}\right) \sin \left[\left(\omega_{\mathrm{opt}} / c\right) x\right]}{\mathcal{L}(\Omega)}\right\} .
\end{aligned}
$$

It is possible to integrate Eq. (C20) exactly, but it is clear that the main contribution will come from mechanical and optical resonances, $\Omega^{\prime}=\Omega_{\mathrm{opt}}$ and $\Omega^{\prime}=\Omega_{m}$. For $\gamma_{m} \ll(1-r) / \tau$ (which is the case for, e.g., fused silica) the major contribution in Eq. $(\mathrm{C} 20)$ is due to the mechanical resonances:

$$
M_{2}(x, \Omega) \sim \frac{D^{2}}{8 \pi^{2}}\left(2 \omega_{\mathrm{opt}} C\right)^{2}\left[K_{1}(x, \Omega)+K_{2}(x, \Omega)+K_{3}(x, \Omega)\right],
$$

where

$$
\begin{gathered}
K_{1}(x, \Omega) \sim \frac{2 \sin ^{2}\left\{\left[\left(\omega_{\mathrm{opt}}-\Omega_{m}\right) / c\right] x\right\} \sin ^{2}\left\{\left[\left(\omega_{\mathrm{opt}}+\Omega_{m}\right) / c\right] x\right\}+\sin ^{4}\left\{\left[\left(\omega_{\mathrm{opt}}+\Omega_{m}\right) / c\right] x\right\}+\sin ^{4}\left\{\left[\left(\omega_{\mathrm{opt}}-\Omega_{m}\right) / c\right] x\right\}}{16 \sin ^{4}\left(\Omega_{m} \tau\right) \Omega_{m}^{4} \Omega^{2} \gamma_{m}}, \\
K_{2}(x, \Omega) \sim \frac{\left(\sin \left\{\left[\left(\omega_{\mathrm{opt}}+\Omega\right) / c\right] x\right\}+\sin \left\{\left[\left(\omega_{\mathrm{opt}}-\Omega\right) / c\right] x\right\}\right)^{2}}{16 \sin ^{2}\left(\Omega_{m} \tau\right)(\Omega \tau)^{2} \Omega_{m}^{4} \Omega^{2} \gamma_{m}}, \\
K_{3}(x, \Omega) \sim \frac{\left(\sin ^{2}\left\{\left[\left(\omega_{\mathrm{opt}}+\Omega_{m}\right) / c\right] x\right\}+\sin ^{2}\left\{\left[\left(\omega_{\mathrm{opt}}-\Omega_{m}\right) / c\right] x\right\}\right) \sin ^{2}\left[\left(\omega_{\mathrm{opt}} / c\right) x\right]}{16 \sin ^{3}\left(\Omega_{m} \tau\right)(\Omega \tau) \Omega_{m}^{4} \Omega^{2} \gamma_{m}} .
\end{gathered}
$$

We are only interested in detection frequencies such that $\Omega \tau \ll 1$. Then for the configuration of nonlinear slabs shown in Fig. 2 the main contribution to the noise in the BK meter readout will come from $K_{2}$ and $K_{3}$. The spectral density of the displacement noise will be

$$
S_{\delta x} \sim \frac{1}{8 \pi^{2}}\left(\frac{k_{B} T_{e}}{m^{*}}\right)^{2} \frac{\omega_{\mathrm{opt}}^{2}}{c^{2}} \frac{\gamma_{m}}{\Omega_{m}^{4} \Omega^{2}(\Omega \tau)^{2}}(\Omega \tau+\epsilon),
$$

where $\epsilon$ characterizes the degree of positioning error and the mismatch of nonlinearities of the two slabs:

$$
\epsilon \sim \max \left(\frac{\left|\chi_{1}^{(3)}\right|-\left|\chi_{2}^{(3)}\right|}{\chi_{1}^{(3)}}, \frac{\delta l}{\lambda}\right) .
$$

Here $\delta l$ is the spatial offset of the central point between the two slabs.

Equation (C26) is the main result of this Appendix. Its implications are discussed at the end of Sec. IV. 
[1] A. Abramovici et al., Science 256, 325 (1992); C. Baradaschia et al., Nucl. Instrum. Methods Phys. Res. A 289, 518 (1990).

[2] K. S. Thorne, in 300 Years of Gravitation, edited by S. W. Hawking and W. Israel (Cambridge University Press, Cambridge, England, 1987), Chap. 9, pp. 430-432.

[3] V. B. Braginsky and F. Ya. Khalili, Phys. Lett. A 218, 167 (1996).

[4] See, e.g., V. B. Braginsky and F. Ya. Khalili, Quantum Mea- surement (Cambridge University Press, Cambridge, England, 1992).

[5] V. B. Braginsky and F. Ya. Khalili, Rev. Mod. Phys. 68, 1 (1996).

[6] M. T. Jaekel and S. Reynaud, Europhys. Lett. 13, 301 (1990); S. P. Vyatchanin and E. A. Zubova, Phys. Lett. A 201, 269 (1995); also an unpublished narrow-band QND scheme by W. Unruh is described in Ref. [2]. 